\title{
Utilização do Mastery Learning em sala de aula: uma abordagem integrada aos Mundos Virtuais
}

\author{
Felipe Becker Nunes ${ }^{1}$, Fabrício Herpich ${ }^{1}$, Manuel Constantino Zunguze ${ }^{1}$, José \\ Valdeni de Lima ${ }^{1}$ \\ ${ }^{1}$ Universidade Federal do Rio Grande do Sul (UFRGS) Av. Paulo Gama, 110, Porto \\ Alegre, RS, Brasil - CEP 90040-060 \\ \{nunesfb, fabricio.herpich, manuelczunguze\}@gmail.com, \\ valdenidinf.ufrgs.br
}

\begin{abstract}
This paper describes the general theory of Mastery Learning, with the proposed use jointly with the Virtual Worlds (OpenSim) to assess student performance. Students of an Accounting Sciences course in a discipline of general informatics learned topics related to the use of HTML. Preliminary results obtained with this proposal were positive and instigators, as well as the difficulties in the application of this research were clarified.
\end{abstract}

Resumo. Este artigo descreve de forma geral a teoria do Mastery Learning, tendo como proposta sua utilização de forma conjunta com os Mundos Virtuais (OpenSim) para averiguar o desempenho dos estudantes. Estudantes do curso de Ciências Contábeis em uma disciplina de informática geral aprenderam tópicos referentes ao uso de HTML. Os resultados preliminares obtidos com esta proposta foram positivos e instigadores, assim como foram esclarecidas as dificuldades existentes na aplicação desta pesquisa.

\section{Introdução}

No cenário tradicional de aprendizagem, cada indivíduo que compõe um grupo de alunos está sujeito às políticas e métodos de aprendizagem propostos pela instituição de ensino e/ou docentes. Um ponto crucial neste tipo de arquitetura funcional está situado no fato de que cada aluno possui características e estilos individuais, visto que não é possível afirmar que cada grupo de alunos irá possuir do mesmo conjunto de particularidades e reagir de forma igualitária às atividades didáticas propostas.

Lin et al. (2013) entendem que para estreitar estas diferenças entre os alunos que atingem percentuais altos e baixos, se configura necessário auxiliar os sujeitos que obtiveram um desempenho inferior ao desejado, por meio de atividades de reforço que levem em consideração as características individuais de cada um. Uma linha de pensamento raciocinada por Bloom (1968) no artigo "Learning for Mastery", converge para a crença de que todos alunos tem o potencial necessário para aprender conteúdos igualmente complexos, ressaltando que alguns poderão despender mais tempo para concluir tal procedimento.

É neste formato de sistema educacional, no qual o que importa é o conteúdo ser ensinado a todos, sendo fornecido mais tempo aos alunos que necessitarem, e, atividades de enriquecimento aos mais avançados, que se encaixa a teoria educacional denominada Mastery Learning. Desta forma, o modelo proposto no Mastery Learning 
defende a crença de que todos os estudantes podem alcançar um nível elevado em seu desempenho acadêmico e suas diferenças de aprendizagem podem ser minimizadas.

Por se tratar de um modelo criado em 1968, aliado ao fato do fenômeno evolucionário ocorrido nas últimas décadas no que concerne à introdução das Tecnologias da Informação e Comunicação (TIC) no âmbito educacional, se torna factível a reflexão sobre o modo de aplicação do Mastery Learning atualmente em sala de aula e a distância utilizando as TIC. Do ponto de vista defendido por Purbohadi et al. (2013), esta teoria pode ser introduzida facilmente tanto no formato presencial quanto na educação a distância, caso o suporte tecnológico seja apropriado.

Diante deste contexto, se torna oportuno introduzir como solução os ambientes virtuais de aprendizagem (Moodle), assim como a proposta do uso dos Mundos Virtuais. Orgaz et al. (2012) explica que os Mundos Virtuais tem como objetivo disponibilizar espaços 3D onde o estudante pode transitar e vivenciar experiências em um ambiente altamente interativo. Conforme os aspectos dissertados anteriormente, que reforçam a interligação das TIC à abordagem Mastery Learning no ensino presencial e a distância, se cria a possibilidade de uma possível integração envolvendo a abordagem de Mundos Virtuais e o emprego de atividades educacionais neste ambiente, baseadas nos preceitos do ML.

Este artigo visa apresentar uma visão geral da aplicação da teoria Mastery Learning no contexto escolar, cuja integração aos Mundos Virtuais é proposta para a realização das atividades educacionais. Para isto, um estudo de caso foi desenvolvido em uma disciplina denominada "Computador e Sistemas de Informação", com o uso do mundo virtual OpenSim e a implementação de diversas atividades educacionais neste ambiente de acordo com os preceitos do Mastery Learning, com o intuito de analisar a viabilidade e os resultados da aplicação desta proposta.

\section{Trabalhos Relacionados}

Ao longo de vários anos, pesquisas têm sido realizadas com base na teoria educacional Mastery Learning, as quais têm variado em termos da população (do ensino básico até ao universitário), e, em alguns casos usando tecnologias educacionais para suporte na realização das atividades. Na literatura podem ser encontrados centenas de trabalhos que utilizam de alguma forma a teoria do ML no decorrer dos últimos 60 anos, em que Leonard, Hollot, \& Gerace (2008) identificaram pelo menos cinco surveys que foram escritos por diferentes pesquisadores neste período, sendo que quatro deles concluíram que grande parte dos trabalhos analisados teve diversos efeitos positivos nas escolas.

Marteleira (2010) explica que o modelo de Bloom teve um grande impacto durante as décadas de 60 e 70, nas quais houve um aumento significativo no número de estudos realizados, se estendendo até o início da década de 80. Entretanto, conforme esclarece Guskey (2010), devido às más interpretações e o surgimento de novas teorias, o ML caiu em esquecimento na década de 80. Corroborando tais asserções, López (2006) também considera que a exigência e algumas dificuldades na operacionalização do ML, como a exigência de um nível elevado de compromisso por parte do docente e a gestão em sala de aula, podem ser considerados como algumas das razões para que o modelo tenha sido progressivamente ignorado, apesar dos seus significativos efeitos na aprendizagem dos alunos. 
As últimas décadas foram marcadas por uma ação de revalorização do Mastery Learning, motivado em grande parte pela evolução tecnológica alavancada pelas TIC e a sua acessibilidade nas escolas, o que cria maiores condições para a implantação deste este tipo de teoria educacional. Com relação à aplicabilidade em diferentes áreas, similarmente aos Mundos Virtuais, está abordagem contempla uma diversificada gama de extensões. Esta afirmação é corroborada por Guskey (2010) em seu trabalho, no qual ele afirma que o ML se mostrou efetivo em uma grande variedade de áreas, como matemática, ciências, língua estrangeira, treinamento e educação médica.

A análise efetuada acerca dos diferentes domínios de aplicação que o Mastery Learning tem sido referenciado e utilizado, demonstra a sua evolução e posterior decadência no decorrer dos anos, assim como a presente recuperação desta teoria educacional, principalmente devido ao uso das TIC, permite esclarecer seu cenário atual. Com base nisso, em relação à sua aplicação nesta proposta, o aporte tecnológico para aplicar esta teoria pode vir a ser preenchido com a utilização dos Mundos Virtuais, o qual possibilita a construção de uma diversificada quantidade de recursos educacionais e atividades didáticas, além da realização de atividades avaliativas, tornando esta proposta diferenciada das demais por utilizar tal ferramenta tecnológica, que dispõe de aspectos de imersividade e objetos tridimensionais.

\section{Mastery Learning}

O ML é um quadro pensado para planejar sequências de instrução com o objetivo de que todos os estudantes possam atingir um nível de desempenho razoável em um determinado conteúdo (MARTELEIRA, 2010). Se caracteriza necessário efetuar de forma detalhada a sua forma de operação e aplicação. Assim, são listados os princípios básicos que norteiam o ML (Kazu et al., 2005).

- Definir claramente os objetivos que representam os propósitos do curso;

- Dividir o currículo em unidades de aprendizagem relativamente pequenas, cada uma com seus próprios objetivos e avaliações;

- Identificar materiais e estratégias de aprendizagem; ensino, modelagem, prática formativa, avaliações, tutorias e reforço devem ser incluídas;

- Cada unidade é precedida por testes de diagnóstico breves (pré-teste) ou avaliações formativas;

- Os resultados dos testes formativos devem ser usados para fornecer instruções suplementares, ou atividades corretivas para ajudar o aluno a superar os problemas.

Portanto, no Mastery Learning os conteúdos são segmentados em pequenas unidades ordenadas por uma sequência lógica com objetivos específicos prédeterminados, que são seguidos até serem alcançados. Os alunos trabalham sozinhos ou em grupos dentro de cada bloco de conteúdo em uma série de etapas sequenciais, nas quais os estudantes devem demonstrar um alto nível de sucesso em testes, geralmente cerca de $80 \%$, antes de avançar para novos conteúdos (MARTELEIRA, 2010).

Os professores procuram evitar a repetição desnecessária de conteúdos, avaliando regularmente os conhecimentos e habilidades. Para isto, um conjunto de 
exames são aplicados ao decorrer de um módulo, no qual deverá ser aplicado um préteste para avaliar a situação inicial de cada indivíduo. Em seguida, um teste avaliativo deve ser aplicado para mensurar se o aluno detém o conhecimento necessário para que possa avançar no módulo ou atividades de reforço devem ser aplicadas.

Os alunos que não atingirem o grau de domínio requisitado, devem receber um feedback imediato com a aplicação de atividades de reforço, enquanto que os demais poderão ser encaixados em atividades de enriquecimento. Para aqueles que não atingem o nível exigido, são fornecidos aulas e/ou conteúdos suplementares, discussões em pequenos grupos, ou lição de casa para que eles possam alcançar o nível esperado. Após terem sido aplicadas as ações corretivas, um novo teste formativo deve ser realizado, a fim de, mensurar o grau de evolução do aluno.

Com relação às desvantagens apontadas, um dos principais pontos criticados no ML está relacionado ao custo de tempo que esta abordagem envolve ao ser aplicada. Docentes e gestores podem considerar inconveniente o tempo adicional que precisa ser fornecido aos estudantes que detêm maiores dificuldades para aprender, devido à grade de horários e formulação de atividades e materiais adicionais.

Entretanto, autores como Guskey (2010) e Marteleira (2010) defendem estas críticas esclarecendo que o tempo adicional necessário não é permanente, visto que Bloom ressaltou em sua proposta que inicialmente os alunos com dificuldades maiores podem necessitar de um tempo maior para o seu aprendizado, mas que no decorrer do curso isto provavelmente iria diminuir com a adequação à abordagem do ML e o aprendizado dos conceitos anteriores que servem como base para novos aprendizados.

Com relação ao maior número de avaliações realizadas nesta teoria, de acordo com Correia et al. (2015), na abordagem tradicional o professor, mesmo quando assessorado por monitores, não consegue fornecer um feedback adequado e rápido para cada uma das soluções propostas pelos alunos, pois a correção de cada solução demanda uma quantidade de tempo considerável. Portanto, se considera necessário que o docente planeje adequadamente as avaliações a serem realizadas e a forma de correção para que o esforço necessário não seja demasiado.

Marteleira (2010) explica que as TIC têm, no que se refere à operacionalização do Mastery Learning, um grande potencial de elaboração de atividades, facilitando assim ao professor o desenvolvimento das unidades de aprendizagem seguindo os princípios e a sequência subjacentes ao ML. Com o uso das TIC no Mastery Learning, atividades que devem ser desenvolvidas pelos alunos podem ser trabalhadas de diferentes maneiras para atender às suas preferências.

\section{Mundos Virtuais}

Griol et al. (2014) tem o entendimento de que os Mundos Virtuais podem ser considerados ambientes gráficos simulados por computador, no qual os seres humanos convivem com outros usuários através de seus avatares. Já Dalsasso et al. (2014) explica que a possibilidade de imersão em ambientes como Second Life e OpenSimulator tem sido utilizada para o desenvolvimento de ambientes colaborativos para uso educacional. Para a visualização e interação com o mundo virtual, é necessária a instalação de uma aplicação conhecida como viewer, no qual o usuário pode interagir e 
realizar suas atividades. De acordo com Nunes et al. (2013), essa aplicação fornece suporte para a visualização, importação e exportação de objetos, e demais recursos relacionados ao mundo virtual.

Johnson et al. (2009) defendem a idéia de que os ambientes imersivos podem oferecer um novo tipo de abordagem, na qual os usuários passam de um estado considerado passivo e observatório para se tornarem sujeitos mais ativos dentro do mundo virtual. Essa linha de pensamento pode ser corroborada pelos diversos recursos presentes neste tipo de ambiente, tendo como exemplo o uso de chat via texto ou voz, navegar pelos cenários dispostos e interagir com os elementos presentes no $\mathrm{MV}$, o que pode gerar um cenário propício para que este tipo de transformação de atitude ocorra.

A criação de ambientes imersivos voltados à educação necessita que diversos fatores sejam considerados, e.g., objetivos pedagógicos e estratégias de ensino bem definidas com base em teorias de aprendizagem, design amigável e objetos capazes de incentivar a interação e colaboração entre os usuários (HERPICH et al., 2014). A natureza imersiva deste tipo de ambiente incentiva os alunos (como avatares) a executarem atividades de aprendizagem que não estavam inicialmente previstas pelos professores, como interagir com outros alunos através de chats, procurar novos conteúdos multimídia ou utilizar novos artefatos físicos 3D para aprender sobre um determinado tópico (FERNÁNDEZ-GALLEGO et al., 2013).

A autonomia fornecida aos estudantes pode auxiliar no desenvolvimento de suas ações próprias e investigações acerca de novos conhecimentos calcados nos recursos presentes nestes ambientes 3D. É importante ressaltar as possibilidades inerentes ao desenvolvimento de atividades de cunho prático e simulações, que demonstrem experimentos que corriqueiramente são difíceis de serem visualizados em laboratórios reais, devido aos custos e/ou perigos intrínsecos à execução destes junto aos alunos.

\section{Metodologia}

A metodologia empregada nesta pesquisa pode ser dividida em diferentes etapas de aplicação, como são detalhadas a seguir. A primeira etapa consistiu em um levantamento teórico acerca dos tópicos Mastery Learning e Mundos Virtuais, assim como foi realizada uma análise do cenário geral e os direcionamentos das pesquisas que envolvem o uso desta teoria educacional.

$\mathrm{Na}$ segunda etapa foi definida a infraestrutura tecnológica utilizada para aporte desta pesquisa. O OpenSim 0.8 .1 foi selecionado por ser gratuito, open source e com uma vasta documentação no meio acadêmico; para a visualização do mundo virtual foi selecionado o viewer Singularity, que possui recursos apropriados para atender às necessidades identificadas na construção do mundo virtual. Para a hospedagem e funcionamento das ferramentas selecionadas usadas, o Wamp Server foi escolhido por ser gratuito e conter os recursos necessários (PHP, MySQL e Apache). Todos os softwares foram instalados em um servidor no qual os clientes acessaram por meio do viewer instalado nos seus computadores.

$\mathrm{Na}$ terceira etapa foi realizado o detalhamento do experimento executado nesta pesquisa, cujo público alvo abrangeu alunos do curso de graduação em Ciências Contábeis da Universidade Federal do Rio Grande do Sul. Um total de 12 participantes 
completaram todas etapas requeridas no experimento, cuja disciplina denominada "Computador e Sistemas de Informação" foi utilizada para a realização dos testes, sendo o experimento composto por dois encontros presenciais.

A formulação das atividades educacionais a serem aplicadas no Mundo Virtual abarcaram o tópico de ensino de recursos multimídias em HTML, que está presente em diferentes áreas de domínio. Desta forma, este tópico foi subdividido em duas unidades para a realização das atividades no mundo virtual, sendo o primeiro voltado para o ensino de inserção de vídeos em páginas $W e b$, e o segundo compreende o estudo acerca da inserção de áudios em páginas Web.

O primeiro encontro presencial ficou caracterizado pela apresentação da proposta, realização do pré-teste e apresentação do mundo virtual aos estudantes para poderem se ambientar e conhecer o modo de funcionamento do ambiente. O segundo encontro presencial foi constituído pelas duas unidades, as quais possuíam atividades de reforço, tanto para os alunos que tiveram um desempenho satisfatório (enriquecimento do conteúdo), como para os alunos que não atingiram a maestria durante sua interação no mundo virtual (reforço do conteúdo), sendo abordada desta forma a ação de feedback por parte do professor junto aos alunos para suprir as dificuldades durante o período de realização das atividades, e, não somente ao final. Após o aluno percorrer as subunidades propostas e atingir a maestria, o mesmo deveria responder ao pós-teste (idêntico ao pré-teste) para avaliação do seu desempenho.

Para o estudo de caso aplicado neste primeiro experimento, um laboratório virtual foi desenvolvido dentro do mundo virtual focalizado no ensino de recursos multimídias em páginas HTML, no qual diferentes tipos de atividades educacionais foram criados, como a apresentação de slides, vídeos, textos, questões e a realização de atividades de cunho prático, como o uso de ferramentas online para a geração de páginas HTML em tempo real.

A quarta etapa abrangeu a revisão preliminar dos resultados obtidos, que foram mensurados tendo como base a teoria Mastery Learning, a qual explicita que para $o$ estudante atingir o nível de maestria exigido, o mesmo deve obter um percentual mínimo, geralmente $80 \%$ na média final do tópico abordado. O desempenho final de cada estudante foi composto pelas notas obtidas ao longo de quatro avaliações realizadas durante este primeiro processo experimental conduzido, sendo os seguintes:

- Pré-teste = possui 9 questões sobre o ensino de recursos multimídias em HTML (vídeo e áudio) para avaliar o conhecimento inicial de cada aluno.

- Avaliação intermediaria 01 = composta por 5 questões pertencentes ao subtópico referente à inserção de vídeos em páginas $W e b$.

- Avaliação intermediaria 02 = composta por 5 questões pertencentes ao subtópico referente à inserção de áudios em páginas $W e b$.

- Pós-teste = este teste será o mesmo realizado no pré-teste, com o intuito de avaliar se ao final do curso o aluno atingiu o nível de maestria exigido na abordagem Mastery Learning.

Desta forma, o desempenho geral de cada estudante por meio das avaliações sugeridas na teoria do Mastery Learning pode ser obtido, e, analisado para ser usado na 
composição das trajetórias de aprendizagem desenvolvidas por cada aluno durante as suas interações com o mundo virtual.

\section{Estudo de Caso}

O experimento desenvolvido contemplou avaliar o desempenho dos alunos que realizaram as atividades educacionais formuladas seguindo os preceitos definidos pelo Mastery Learning, dentro do mundo virtual. No primeiro encontro com a turma, o préteste composto por 9 questões relacionadas aos tópicos de inserção de vídeos e áudios em páginas HTML foi aplicado junto ao grupo de alunos presentes.

Como atividade inicial foi proposto utilizar o OpenSim com o intuito de proporcionar uma fluência destes estudantes com mundo virtual. Entretanto, a adaptabilidade por parte dos 12 alunos ocorreu naturalmente, em um processo que pôde ser considerado avançado, visto que pouquíssimas dúvidas surgiram sobre a forma de utilização do ambiente e do avatar. Findado o processo de ambientação dos alunos ao OpenSim, o docente realizou a descrição do experimento e atividades que seriam desempenhadas por eles, tomando o cuidado de esclarecer os objetivos de cada tópico e da proposta em geral, assim como funcionariam as avaliações no decorrer desta etapa.

Foram desenvolvidos no mundo virtual quatro laboratórios para atender os requisitos exigidos pela abordagem Mastery Learning, sendo os dois primeiros laboratórios, denominados respectivamente de "Laboratório 01" e "Laboratório 01 Reforço", o foco dos materiais estava centrado no ensino da unidade 01 (vídeos no HTML). Os demais laboratórios ("Laboratório 02" e "Laboratório 02 - Reforço") tiveram seu foco situado no ensino da unidade 02 (áudios no HTML).

A figura 1 apresenta a sala de slides, na qual os usuários podiam efetuar a leitura dos conteúdos trabalhados no tópico. Já na figura 2 é demonstrada a interação dos usuários com a ferramenta "Online HTML Editor", disponibiliza diretamente no mundo virtual e que fornecia em tempo real o resultado dos códigos criados.
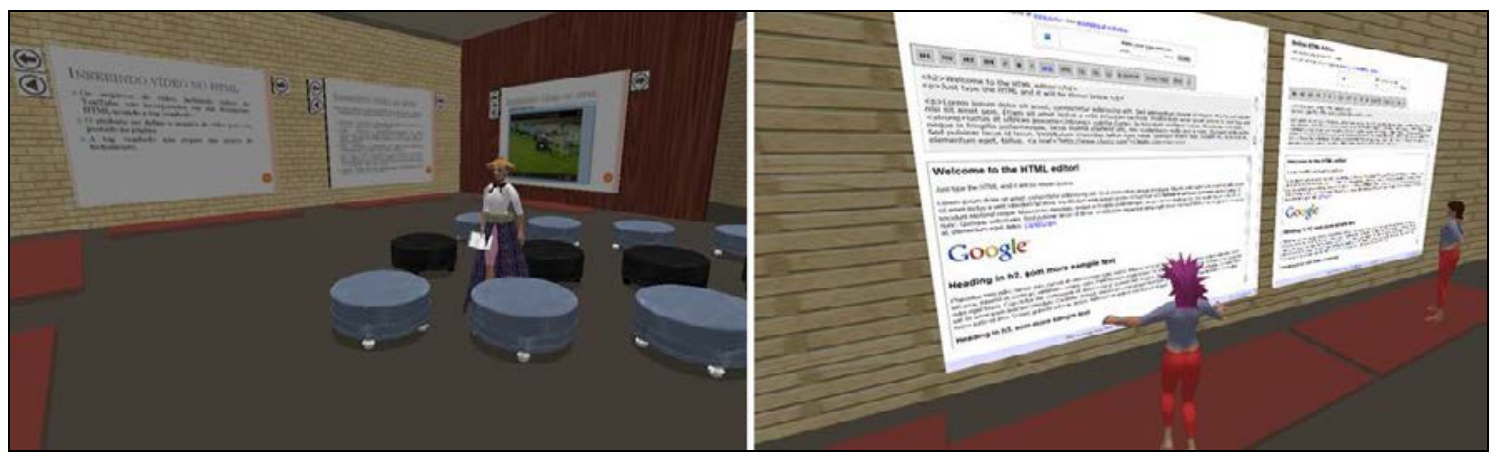

Figura 1 e 2. Sala de slides e Sala com a ferramenta online de HTML

O início das atividades ocorreu no Laboratório 01 com a participação de todos os usuários, que adotaram uma postura ativa e independente, o que resultou em uma fácil utilização dos conteúdos dispostos no laboratório e a existência de um número pequeno de dúvidas durante a interação. O primeiro encontro contou com a realização de um teste intermediário composto por 5 questões relacionadas ao conteúdo discorrido, para avaliar o desempenho dos estudantes após realizarem as interações com os materiais no Mundo Virtual. 
As avaliações foram corrigidas e aqueles alunos que não obtiveram uma nota superior à $80 \%$ foram guiados para o Laboratório 01 - Reforço, com o objetivo de realizar atividades complementares, e os alunos que atingiram a maestria estavam liberados para transitar neste laboratório para ler novos conteúdos e interagir, no intuito de agregar um enriquecimento maior a seu conhecimento. Para finalizar a primeira unidade, os alunos que não haviam atingido a média, realizaram o teste avaliativo novamente, com o objetivo de mensurar o seu desempenho após a realização das atividades de reforço.

Na segunda unidade, as atividades ocorreram no Laboratório 02, que contou com a participação ativa e caracterizada pela motivação dos alunos em utilizarem o ambiente novamente para aprender os conteúdos abordados. Nesta segunda unidade, o tema de inserção de áudios em HTML foi estudado pelos alunos no laboratório, que seguia exatamente o mesmo formato e estrutura utilizada no primeiro encontro.

Após terem interagido com os recursos disponibilizados nas cinco salas do laboratório, os alunos foram orientados a responderem a um teste intermediário composto por 5 questões relacionadas ao conteúdo discorrido, para avaliar o seu desempenho. Passada a aplicação do teste, as avaliações foram corrigidas e aqueles alunos que não obtiveram uma nota superior à $80 \%$ foram guiados para o Laboratório 02 - Reforço, com o objetivo de realizarem atividades complementares, e os alunos que atingiram o grau de maestria estavam liberados para transitar neste laboratório para ler novos conteúdos, no intuito de agregar um enriquecimento maior a seu conhecimento.

Os alunos que não haviam atingido a nota realizaram o teste avaliativo novamente, com o objetivo de mensurar o seu desempenho após a realização das atividades de reforço. Para finalizar o segundo encontro, o pós-teste foi respondido por eles com o objetivo de fornecer um comparativo de desempenho entre o conhecimento inicial que detinham antes de iniciar a aprendizagem das duas unidades, em relação aos novos conceitos adquiridos ao término dos dois encontros.

Com relação aos resultados obtidos nas avaliações, a figura 3 apresenta a média dos alunos em cada avaliação. Relacionando primeiramente os resultados do pré-teste com os pós-teste, notadamente observa-se uma evolução nas notas obtidas, sendo que no primeiro teste o intervalo ficou entre 45 e 89 , enquanto que no último teste as notas variaram de 78 a 100.

No que diz respeito às notas dos testes intermediários, na unidade 01 a média resultante foi positiva $(87,14)$, tanto no primeiro teste quanto na recuperação, da qual participaram três alunos. Dos 12 estudantes, três realizaram o reforço e obtiveram uma evolução significativa com uma nota de 93,3, praticamente atingindo a média geral dos que não estavam em recuperação. Na unidade 02 , a média geral $(75,71)$ ficou abaixo do esperado devido a concentração de notas no percentual de $80 \%$ e também das quatro notas abaixo, que ficaram entre 20 e 60 , diminuindo a média geral. Os quatro alunos que necessitaram de reforço, assim como na unidade 01 , demonstraram uma evolução e obtiveram uma média positiva (90). 


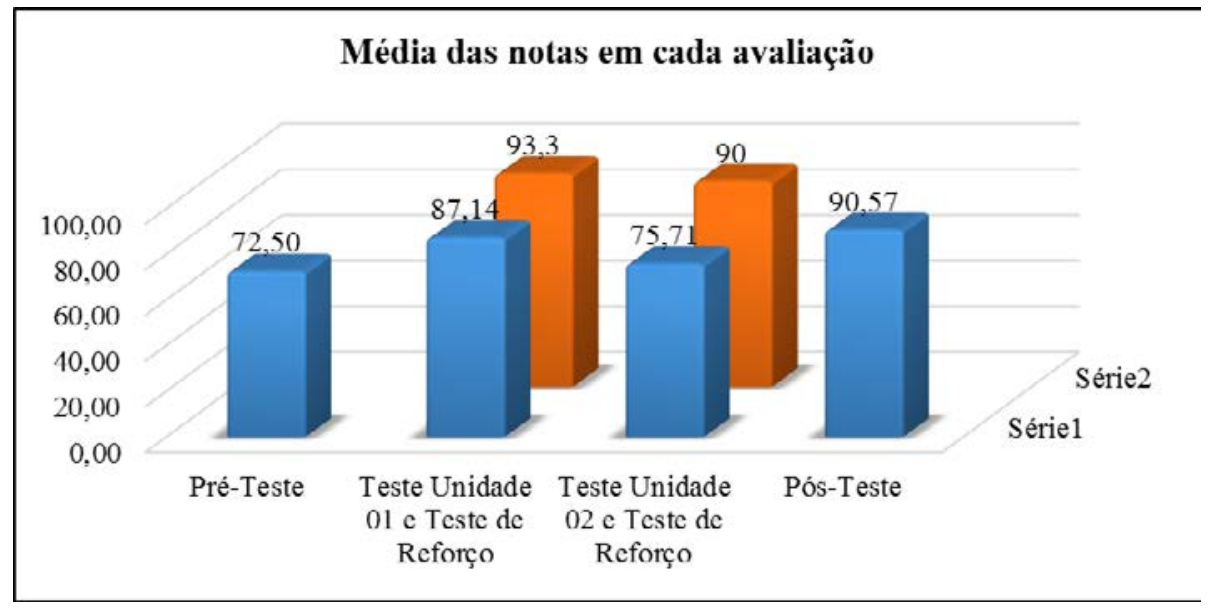

Figura 3. Resultados gerais das avaliações realizadas pelos participantes

Desta forma, é possível notar o progresso nos resultados obtidos nos testes realizados, demonstrando que poucos alunos necessitaram realizar a recuperação, e, aqueles que a efetuaram obtiveram notas iguais ou superiores à média da turma dos aprovados. Associado a isto, o desempenho crescente evidenciado no gráfico, tomando como comparativo o pré-teste e o pós-teste, provê indícios positivos de que a abordagem envolvendo Mundos Virtuais e Mastery Learning operou de forma satisfatória.

\section{Considerações Finais}

O uso do Mastery Learning no contexto escolar tem sido retomado no decorrer dos últimos anos, gerando resultados positivos no que concerne ao desempenho dos alunos. É importante ressaltar que a sua metodologia de aplicação requer um esforço maior por parte do professor, assim como demanda um tempo extra.

Nesta presente proposta, que está voltada para a aplicação do Mastery Learning utilizando os Mundos Virtuais como aporte tecnológico, por meio da análise dos resultados preliminares deste estudo foi possível identificar notar indícios positivos na melhoria de desempenho dos alunos. As atividades de reforço aplicadas e de especialização podem ser consideradas como um dos principais elementos que interferiram para que houvesse esta melhoria, assim como, as constantes avaliações que reproduzem para o docente o cenário geral e individual da turma.

Apesar das melhorias, o tempo disposto para a realização das atividades educacionais foi mais extenso do que o utilizado corriqueiramente em uma aula tradicional, o que pode ser considerado um fator negativo devido às exigências presentes em uma instituição de ensino quanto aos horários. Como abordagem futura, está a realização de testes mais robustos, levando em consideração um semestre ou mais, assim como a apresentação de uma alternativa à problemática do tempo, que está focada na realização das atividades de reforço e enriquecimento fora do período normal de aula.

\section{Referências}

BLOOM, B. S. Learning for Mastery. Regional Education Laboratory for the Carolinas and Virginia, Topical Papers and Reprints, v. 1, n. 2, 1968.

CORREIA, A. L.; COSTA, D. V. B. DA; BARBOSA, A. DE A.; COSTA, E. DE B. 
Uso de avaliação por pares em disciplinas introdutórias de programação. Workshop sobre Educação em Computação, p.1-10, 2015.

DALSASSO, P.; BOS, A. S.; BERNARDI, G.; MÜLLER, F. M.; CORDENONSI, A. Z. Resgatando A História da Computação através de um Museu Virtual 3D Interativo. Workshop sobre Educação em Computação, p.1-10, 2014.

FERNÁNDEZ-GALLEGO, B.; LAMA, M.; VIDAL, J. C.; MUCIENTES, M. Learning analytics framework for educational virtual worlds. Procedia Computer Science, v. 25, p. 443-447, 2013.

GRIOL, D.; MOLINA, J.; CALLEJAS, Z. An approach to develop intelligent learning environments by means of immersive virtual worlds. Journal of Ambient Intelligence and Smart Environments, v. 6, n. 2, p. 237-255, 2014.

GUSKEY, T. Lessons of Mastery Learning. Educational Leadership, v. 2, n. 68, p. 52-57, 2010.

HERPICH, F.; NUNES, F. B.; VOSS, G. B.; JARDIM, R. R.; MEDINA, R. D. Ambiente Virtual Imersivo para ensino em Redes de Computadores: uma proposta usando Agentes Inteligentes. $25^{\circ}$ Simpósio Brasileiro de Informática na Educação (SBIE) - $3^{\circ}$ Congresso Brasileiro de Informática na Educação (CBIE), p.65-69, 2014.

JOHNSON, C. M.; VORDERSTRASSE, A.; SHAW, R. Virtual Worlds in Health Care Higher Education. Journal of Virtual Worlds Research, v. 2, n. 2, p. 1-12, 2009.

KAZU, I. Y.; KAZU, H.; OZDEMIR, O. The Effects of Mastery Learning Model on the Success of the Students Who Attended "Usage of Basic Information Technologies" Course. Educational Technology \& Society, v. 8, n. 4, p. 233-243, 2005.

LEONARD, W. J.; HOLLOT, C. V.; GERACE, W. J. Mastering Circuit Analysis: An innovative approach to a foundational sequence. 38th ASEE/IEEE Frontiers in Education Conference, p.1-6, 2008.

LIN, C. H.; LIU, E. Z.; CHEN, Y.; et al. Game-Based Remedial Instruction in Mastery Learning for Upper-Primary School Students. Educational Technology \& Society, v. 16, n. 2, p. 271-281, 2013.

LÓPEZ, E. El mastery learning a la luz de la investigación educativa. Revista Educación, v. 340, p. 625-665, 2006.

MARTELEIRA, C. P. Mastery Learning - a revalorização de um modelo de ensinoaprendizagem em cursos profissionais?, Dissertação de Mestrado, Portugal, 2010.

NUNES, F. B.; VOSS, G. B.; HERPICH, F.; et al. Viewers para ambientes virtuais imersivos: uma análise comparativa teórico-prática. RENOTE - Revista Novas Tecnologias na Educação, v. 11, n. 1, p. 1-10, 2013.

ORGAZ, G. B.; R-MORENO, M. D.; CAMACHO, D.; BARRERO, D. F. Clustering avatars behaviours from virtual worlds interactions. Proceedings of the 4th International Workshop on Web Intelligence \& Communities, 2012.

PURBOHADI, D.; NUGROHO, L.; SANTOSA, I.; KUMARA, A. GaMa Feedback Learning Model: Basic Concept and Design. Journal of e-Learning and Knowledge Society, v. 9, n. 3, p. 67-77, 2013. 